\title{
Goldenhar syndrome: clinical features with orofacial emphasis
}

\author{
Hercílio MARTELLI-JÚNIOR ${ }^{1}$, Roseli Teixeira de MIRANDA ${ }^{2}$, Cassandro Moreira FERNANDES $^{3}$, \\ Paulo Rogério Ferreti BONAN ${ }^{1}$, Lívia Máris Ribeiro PARANAÍBA ${ }^{4}$, Edgard GRANER ${ }^{5}$, Ricardo D. COLETTA ${ }^{5}$
}

1- DDS, PhD, Stomatology Clinic, Dental School, State University of Montes Claros, Montes Claros, MG, Brazil.
2- DDS, PhD, Dental School, University of Alfenas, Alfenas, MG, Brazil.
3- DMD, Medical School, University of Alfenas, Alfenas, MG, Brazil.
4- DDS, MSc, Department of Oral Diagnosis, Dental School, State University of Campinas, Piracicaba, SP, Brazil.
5- DDS, PhD, Department of Oral Diagnosis, Dental School, State University of Campinas, Piracicaba, SP, Brazil.

Corresponding address: Paulo Rogério Ferreti Bonan - Clínica de Estomatologia - Curso de Odontologia, Universidade Estadual de Montes Claros - Campus Universitário “Darcy Ribeiro” - 39401-089 - Montes Claros, MG - Brazil - e-mail: pbonan@yahoo.com

Received: March 18, 2009 - Modification: September 07, 2009 - Accepted: September 16, 2009

\section{ABSTRACT}

bjectives: Goldenhar syndrome (GS) is a relatively common developmental disorder characterized by craniofacial anomalies in association with vertebral, cardiac, renal, and central nervous system defects. This paper describes GS features with special emphasis on oral characteristics. Material and Methods: The clinical features of 6 patients with GS aged 3 months to 12 years are described, and a brief review of the literature about this genetic disorder is presented. Results: All patients demonstrated the classical triad of GS, including mandibular hypoplasia resulting in facial asymmetry, ear and/or eye malformation, and vertebral anomalies. In addition, renal and gastrointestinal abnormalities were observed in 2 patients. Regarding the oral involvement, 2 patients presented cleft lip and palate, and 1 patient had temporomandibular joint malformation. Malocclusion was found in all patients. Conclusion: Our orofacial findings correlate with the reported cases in the literature, and point out that after diagnosis GS patients need to be examined for systemic abnormalities.

Key words: Goldenhar syndrome. Mandible. Facial asymmetry.

\section{INTRODUCTION}

Goldenhar syndrome (GS), also known as oculoauriculovertebral dysplasia or hemifacial microsomia has a wide range of clinical manifestations, including craniofacial, vertebral, cardiac, renal, and central nervous system anomalies ${ }^{1}$. The typical presentation of GS includes epibulbar dermoids, microtia, mandibular hypoplasia, and vertebral anomalies ${ }^{8,10-11}$. The classic facial aspect of GS patients, described as hemifacial microsomia, and the other anomalies of this syndrome are probably caused by development defects of the first and second brachial archs ${ }^{12}$. The causes for these developmental defects seem to be heterogeneous ${ }^{2}$.

GS is a condition with a prevalence ranging from $1: 3,500$ to $1: 7,000$ live births, and a male-female ratio of $3: 2^{4}$. Although most cases are sporadic, familial occurrences have been observed ${ }^{8}$. Oral manifestations of GS are clearly heterogeneous, and range from malocclusion to a more complex phenotype with complete absence of the mandibular ramus and temporomandibular joint (TMJ) $)^{3,13}$. Different forms of cleft lip and palate and decreased palatal width are frequently found in GS patients ${ }^{4}$. This paper describes the clinical features of GS, with emphasis on the oral manifestations, by presenting the cases of 6 patients and briefly reviewing the literature about this genetic disorder.

\section{MATERIAL AND METHODS}

Six cases of GS were retrospectively reviewed. The patients were diagnosed between 2004 and 2007 at the Center for Rehabilitation of Craniofacial Anomalies of the Dental School of the University of Alfenas, Brazil. The diagnosis was established according to the minimal diagnostic criteria proposed by Stromland, et al. ${ }^{9}$ (2007), considering the combination of hemifacial microsomia, vertebral anomaly and ear or eye malformation. From the patient files, we collected the following data: age, gender, maternal and paternal age at pregnancy, 
Figure 1- The main clinical findings of the 6 patients with GS

\begin{tabular}{|c|c|c|c|c|c|c|}
\hline Clinical findings & Patient 1 & Patient 2 & Patient 3 & Patient 4 & Patient 5 & Patient 6 \\
\hline \multicolumn{7}{|l|}{ Craniofacial Features } \\
\hline Mandibular hypoplasia & + & + & + & + & + & + \\
\hline Facial asymmetry & + & + & + & + & + & + \\
\hline Cleft lip and palate & - & - & + & - & + & - \\
\hline Narrowed palate & - & + & - & - & - & - \\
\hline TMJ malformation & - & - & - & - & - & + \\
\hline Malocclusion & + & + & + & + & + & + \\
\hline \multicolumn{7}{|l|}{ Ear abnormalities } \\
\hline Anotia & - & - & - & - & - & + \\
\hline Microtia & + & - & + & - & - & + \\
\hline Preauricular tag & + & + & + & + & - & - \\
\hline \multicolumn{7}{|l|}{ Eye abnormalities } \\
\hline Microphthalmia & + & + & - & - & + & - \\
\hline Anophthalmia & + & - & - & - & - & - \\
\hline Epibulbar dermoid tumor & - & + & + & - & - & - \\
\hline Eyelid coloboma & + & - & - & - & - & - \\
\hline \multicolumn{7}{|l|}{ Others } \\
\hline Vertebral abnormality & + & + & + & + & + & + \\
\hline Cardiac abnormality & - & - & - & - & - & - \\
\hline Renal abnormality & + & - & - & - & - & + \\
\hline Gastrointestinal abnormality & - & - & - & - & - & + \\
\hline
\end{tabular}

(+) presence and (-) absence. TMJ: temporomandibular joint.

information on prenatal and perinatal periods, and family history. All patients were submitted to clinical evaluation, which included general and craniofacial examination. Skull, facial and thoracic radiographs, electrocardiogram and abdominal ultrasonography scans were also obtained. Informed consent was taken from the legal guardians of the children, and the study was approved by Ethics Committee of the Dental School of the University of Alfenas.

\section{RESULTS}

Five patients were females and 1 was male, and the mean age at diagnosis was 7.15 years (ranging from 3 months to 12 years). The mean maternal age was 26.3 years, and the mean paternal age was 32.2 years. There was no history of exposure neither to known teratogenic agents nor maternal diseases. All pregnancies were described as normal, and perinatal complications were not mentioned in any case. All cases were isolated and no parental consanguinity was observed. When applicable, patients demonstrated age-appropriate mental and speech development.

The main clinical findings of the GS patients are depicted in Figure 1. All patients showed mandibular hypoplasia, facial asymmetry and vertebral anomalies resulting in marked scoliosis and/or kyphosis (Figure 2). Ear abnormalities were observed in all patients, except for patient \#5. One (16.6\%) patient showed anotia, 3 (50\%) presented microtia, and preauricular tags were observed in $4(66.6 \%)$ patients (Figures 3 A-B). Regarding
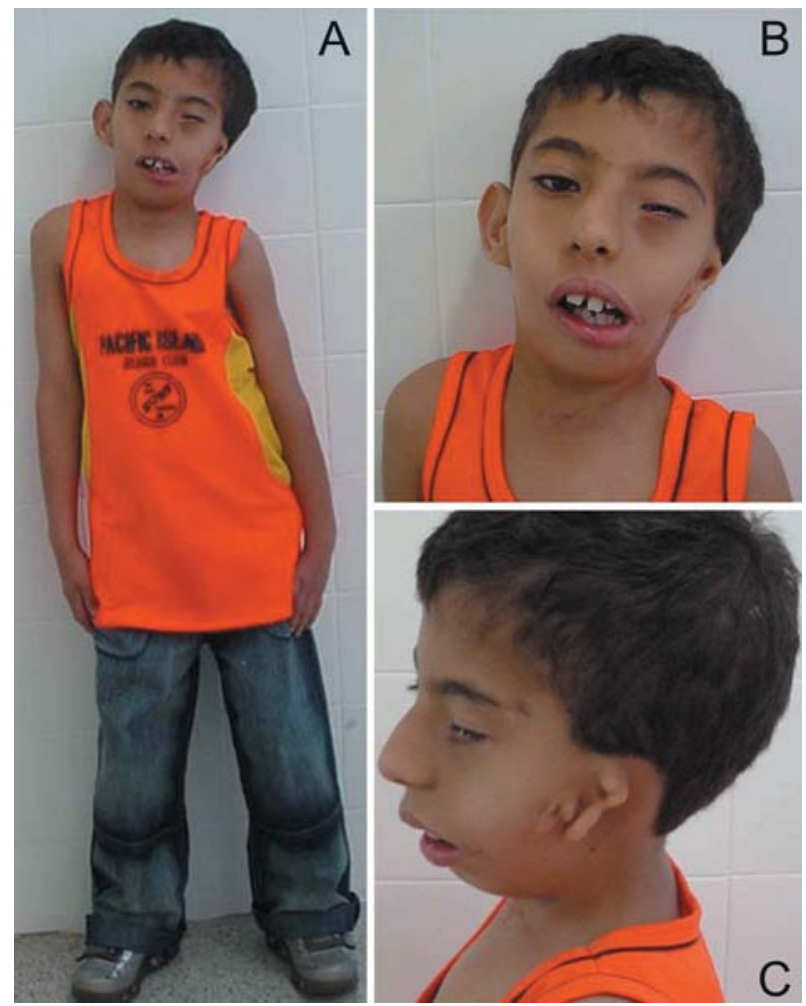

Figure 2- Skeletal and facial phenotypes of patient \#2 of this study. (A) Anterior standing photograph of the patient, demonstrating severe lordoscoliosis. (B) Frontal view of the face showing marked facial asymmetry, malocclusion, and eye involvement characterized by microphthalmia and eyelid coloboma of the left eye. (C) Lateral view of the face showing ear malformations, including microtia and preauricular tags (The parents signed informed consent authorizing the publication of these pictures) 


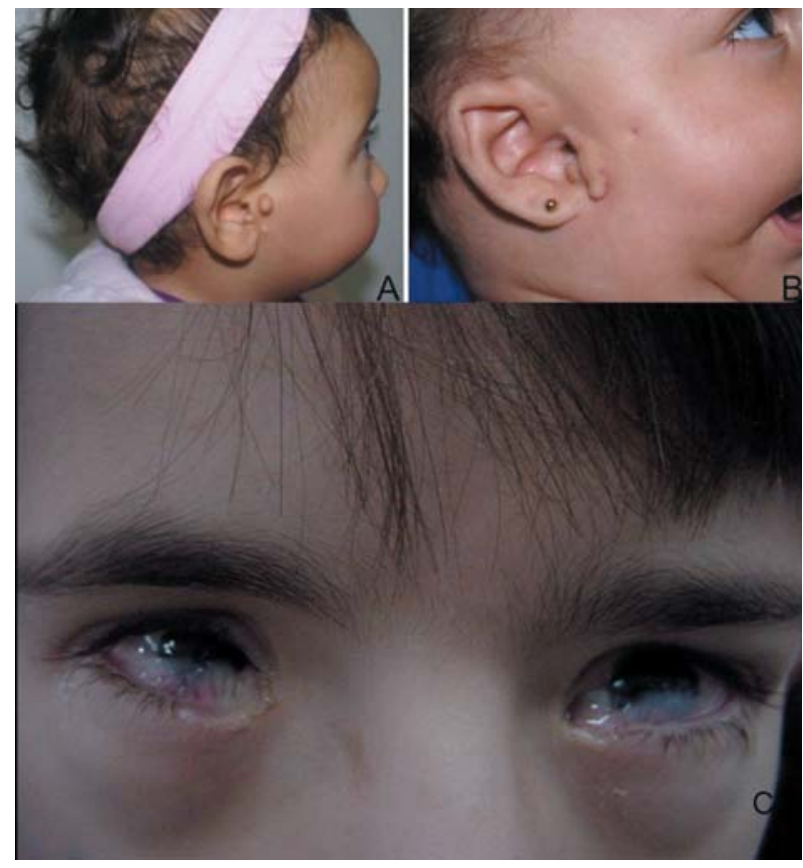

Figure 3- A-B Presence of preauricular tags in patients \#1 (A) (multiple accessory tags) and \#4 (B) of this study. Preauricular tags are considered the most common malformation in GS, and the identification should lead to the search of other GS-associated abnormalities. (C) Bilateral epibulbar dermoid tumors affecting the patient \#3 of this study (The parents signed informed consent authorizing the publication of these pictures)

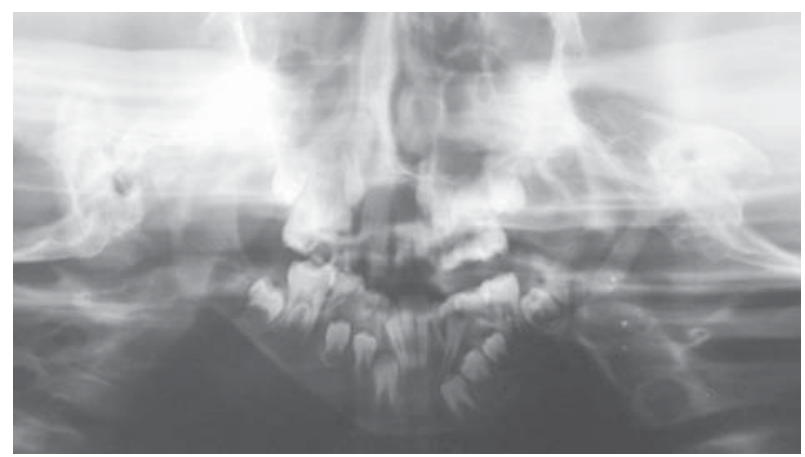

Figure 4- Panoramic radiograph of patient \#6 of this study. The patient showed severe mandibular hypoplasia on the left side associated with hypoplastic temporomandibular joint and marked malocclusion (The parents signed informed consent authorizing the publication of these pictures)

eye abnormalities, $50 \%$ of the patients showed microphthalmia, 2 (33.3\%) showed epibulbar dermoid tumors (Figure $3 \mathrm{C}$ ), and anophthalmia and eyelid coloboma were observed in the patient 1 . Malocclusion was found in all patients, whereas cleft lip and palate was observed in 2 (33.3\%) patients, and narrowed palate and temporomandibular joint (TMJ) malformation (Figure 4) were observed in 1 patient each.
Genetic counseling was given to all parents regarding the inheritance patterns, recurrence risks, possible ways of expression, and the consequences of each phenotype. The patients are currently on medical and regular dental treatment and under observation for other possible malfunctions, including mental retardation, cardiac problems and hearing-speaking difficulties.

\section{DISCUSSION}

Several terms are used to describe GS due to the wide spectrum of anomalies that may be associated with this condition. The authors suggested the use of term oculoauriculovertebral dysplasia to describe the syndrome characterized by epibulbar dermoids and/or lipodermoids, auricular appendages, auricular fistulas, and vertebral anomalies ${ }^{1,8,11}$. However, an overlap in the clinical features of GS, hemifacial microsomia, and oculoauriculovertebral dysplasia and suggested the term oculo-auriculovertebral (OAV) spectrum to describe the patients affected by this association. Although OAV spectrum term is more inclusive and descriptive, GS is more widely recognized and traditionally used.

The etiology of GS remains unknown. Recently, Hartsfield $^{2}$ (2007) reviewed the literature and suggested that GS is resulted of some type of vascular perturbation and/or neural crestopathy during a critical time of embryogenesis. Although autosomal dominant, autosomal recessive and multifactorial inheritance patterns have been reported in the literature, most of GS cases are sporadic.

GS is clinically heterogeneous, and there is no agreement in the literature on the minimal diagnostic criteria. Traditionally, the presence of ear abnormalities, which leads to the search of mandibular hypoplasia and vertebral alterations, is the main clinical feature associated with GS diagnosis $^{9}$. However, the absence of evident features or the unknowledge of GS characteristics make the diagnosis difficult and late. Although all patients were examined and treated by several doctors before our examination, only 2 patients were prematurely diagnosed at ages of 3 and 8 months. The classical features of GS patients involve ocular anomalies, including microphthalmia, anophthalmia, epibulbar dermoid (or lipodermoid) tumors, and eyelid colobomas, aural defects, such as preauricular tags, anotia, microtia and hearing loss, vertebral abnormalities, such as scoliosis, hemivertebrae and cervical fusion, and mandibular hypoplasia ${ }^{8-12}$. Facial involvement is usually unilateral, resulting in a marked asymmetry. In the series presented here the patients were affected unilaterally, and all showed mandibular hypoplasia and vertebral anomaly. Mandibular hypoplasia 
resulted in facial asymmetry in all patients, with 3 showing severe features of hemifacial microsomia. Interestingly, facial asymmetry is reported in 65$75 \%$ of cases of $\mathrm{GS}^{10}$. We also observed an overall frequency of ear anomalies in $83 \%$ and a frequency of eye malformations in $66 \%$ of patients. Ear abnormalities, ranging from anotia to preauricular tag, are considered the most common malformation in GS, with an incidence of $100 \%$ of cases ${ }^{10}$. Interestingly, the patient 5 did not show any ear involvement, but presented enough clinical features to fulfill the diagnosis of GS, including mandibular hypoplasia, scoliosis, microphthalmia and cleft lip and palate. Cleft lip and palate is observed in $7-25 \%$ of GS patients ${ }^{10}$, compared to $34 \%$ in our studied patients.

Early diagnosis of GS is essential for an appropriate treatment of the affected patients and genetic counseling. GS spectrum of malformations includes many other pathological findings, regarding the cardiovascular, pulmonary, central nervous, and gastrointestinal systems ${ }^{9-10}$. After GS diagnosis, our patients were examined by specialists that identified renal alterations in 2 patients and gastrointestinal abnormality in 1 patient. Although the frequency of cardiovascular alterations and conductive hearing loss has been reported to occur with an overall frequency of approximately $50 \%{ }^{11}$, in this case series, no cardiovascular alterations or deafness was found. Recently, Tasse, et al.10 (2005) reported that anomalies of the eye or orofacial clefts in GS patients are predictive of brain malformations. Although 4 of our patients presented eye abnormalities or cleft lip and palate, central nervous system alterations were not observed.

Syndromes derived from aberrations in the development of the first and second branchial arches are in the spectrum of GS, including Treacher-Collins syndrome (TCS). The presence of facial asymmetry and far less hypoplasia of the malar bones in GS are important features to differentiate it from TCS ${ }^{6}$. The TCS affected patients presented downward slating palpebral fissures, colobomas, zygomatic and mandibular hypoplasia, partial absence of the lower eyelid cilia, and abnormalities of the ears ${ }^{6}$.

In summary, the molecular basis of GS is still unclear, and currently no specific diagnostic test is available. Patients with GS can have multiple congenital anomalies, and they need particular attention to internal abnormalities. Pediatric specialists should consult with ear-nose-throat, orthopedics, neurosurgery, and ophthalmology clinics to decide on the most appropriate treatment plan, which varies with age and systemic associations. Dental care with experienced multidisciplinary team of orthodontists and maxillofacial surgeons is also necessary for good results ${ }^{5,7}$. Treatment of patients with mandibular aplasia is complex and can be made with rib grafts, whereas underdeveloped mandibles can be lengthened with bone distraction devices ${ }^{5,7}$. The management of GS patients requests a long-term commitment, and involves multiple procedures spanning the child's period of growth and development.

\section{ACKNOWLEDGMENTS}

This work was partially supported by grants from the State of Minas Gerais Research FoundationFAPEMIG, and the National Council for Scientific and Technological Development-CNPq.

\section{REFERENCES}

1- Anderson PJ, David DJ. Spinal anomalies in Goldenhar Syndrome. Cleft Palate Craniofac J. 2005;42:477-80.

2- Hartsfield JK. Review of the etiologic heterogeneity of the oculo-auriculo-vertebral spectrum (Hemifacial Microsomia). Orthod Craniofac Res. 2007;10:121-8.

3- Kaneyama K, Segami N, Hatta T. Congenital deformities and developmental abnormalities of the mandibular condyle in the temporomandibular joint. Congenit Anom (Kyoto). 2008;48:11825.

4- Kokavec R. Goldenhar syndrome with various clinical manifestations. Cleft Palate Craniofac J. 2006;43:628-34.

5- Lima MD, Marques YM, Alves SM Jr, Ortega KL, Soares MM, Magalhães MH. Distraction osteogenesis in Goldenhar Syndrome: case report and 8-year follow-up. Med Oral Patol Oral Cir Bucal. 2007;12:E528-31.

6- Martelli-Júnior H, Coletta RD, Miranda RT, Barros LM, Swerts MS, Bonan PR. Orofacial features of Treacher Collins syndrome. Med Oral Patol Oral Cir Bucal. 2009;14(7):E344-8.

7- Montoya Pérez LA, Arenas Sordo ML, Hernández Zamora E, Aldape Barrios BC. Oral pathology in a group of Mexican patients with genetic diseases. Med Oral Patol Oral Cir Bucal. 2007;12:E92-5.

8- Rollnick BR, Kaye CI, Nagatoshi K, Hauck W, Martin AO. Oculoauriculovertebral dysplasia and variants: phenotypic characteristics of 294 patients. Am J Med Genet. 1987;26:361-75. 9- Stromland K, Miller M, Sjogreen L, Johansson M, Joelsson BM, Billstedt $E$, et al. Oculo-auriculo-vertebral spectrum: associated anomalies, functional deficits and possible developmental risk factors. Am J Med Genet A. 2007;143A:1317-25.

10- Tasse C, Bohringer S, Fischer S, Ludecke HJ, Albrecht B, Horn D, et al. Oculo-auriculo-vertebral spectrum (OAVS): clinical evaluation and severity scoring of 53 patients and proposal for a new classification. Eur J Med Genet. 2005;48:397-411.

11- Touliatou V, Fryssira H, Mavrou A, Kanavakis E, Kitsiou-Tzeli S. Clinical manifestations in 17 Greek patients with Goldenhar syndrome. Genet Couns. 2006;17:359-70.

12- Vendramini S, Richieri-Costa A, Guion-Almeida ML. Oculoauriculovertebral spectrum with radial defects: a new syndrome or an extension of the oculoauriculovertebral spectrum? Report of fourteen Brazilian cases and review of the literature. Eur J Hum Genet. 2007;15:411-21.

13- Vilkki SK, Hukki J, Nietosvaara Y, Hurmerinta K, Suominen E. Microvascular temporomandibular joint and mandibular ramus reconstruction in hemifacial microsomia. J Craniofac Surg. 2002;13:809-15. 